\title{
Análisis comparativo del funcionamiento del inyector de un motor CRDI bajo diferentes alturas geográficas
}

\section{Comparative analysis of the operation of a CRDI motor injector under different geographic heights}

Juan José Castro Mediavilla

Marcelo Eduardo González Torres

Universidad Internacional del Ecuador, Ecuador

Autor para correspondencia: jucastrome@uide.edu.ec, magonzalezto@uide.edu.ec Fecha de recepción: 01 de agosto de 2018 - Fecha de aceptación: 01 de diciembre de 2018

Resumen: La presente investigación inicia indicando los sistemas de inyección diésel convencional y los sistemas de inyección diésel riel común, destacando las ventajas de disminuir el consumo de combustible, el ruido y la reducción de gases contaminantes al medio ambiente. En la parte teórica se describe la composición de los sistemas de combustible de riel común, los diferentes componentes, los actuadores que se analizaron durante las pruebas tanto en la altura como a nivel del mar. Posteriormente se determinó el ciclo de experimentación ajustado a la investigación realizada, se estableció el protocolo de pruebas, ciclo de manejo y lugares a realizar las pruebas. Se muestra el vehículo de pruebas, los equipos de medición que van a ser utilizados y posteriormente se realizan las mediciones con el osciloscopio para la recopilación de datos de las diferentes pruebas realizadas tanto estáticas como dinámicas. El análisis de resultados de las pruebas estáticas muestra datos interesantes sobre el comportamiento del vehículo tanto en la altura como a nivel del mar. El detalle de los oscilogramas nos permite comprender de manera clara el funcionamiento de los actuadores del sistema riel común.

Palabras Clave: inyección; común; riel; presión

Abstract: The present investigation begins by indicating the systems of conventional diesel injection and common rail diesel injection systems, highlighting the advantages of reducing fuel consumption, noise and the reduction of polluting gases to the environment. The theoretical part describes the composition of the common rail fuel systems, the different components, the actuators that were analyzed during the tests both at sea level and at sea level. Subsequently, the experimentation cycle adjusted to the research carried out was determined, the test protocol was established, the driving cycle and places to perform the tests. The test vehicle is shown, the measurement equipment that will be used and subsequently the measurements are made with the oscilloscope for the data collection of the different tests carried out both static and dynamic. The analysis of the results of the static tests shows interesting data about the behavior of the vehicle both in the height and at sea level. The detail of the oscillograms allows us to understand clearly the operation of the actuators of the common rail system.

Key words: injection; common; rail; pressure 


\section{Introducción}

El avance de la electrónica está presente en muchas áreas y aplicaciones de la vida moderna, en el campo automotriz no es la excepción. El vertiginoso avance tecnológico permite llegar de una forma casi certera y eficiente a las distintas fallas de los vehículos, el osciloscopio es una de las herramientas que permite una gran versatilidad en el diagnóstico, ya que permite graficar en tiempo real el comportamiento de una señal u orden, es decir no solo brinda un parámetro numérico sino también gráfico. (Van \& Meyer, 1981).

En base a escalas usadas en el osciloscopio se obtienen señales que son fáciles de interpretar, estas a su vez se las puede analizar, grabar, transferir y registrar.

La capacidad de análisis se multiplica y el tiempo de diagnóstico se reduce, siendo más efectivo y en menor tiempo. Los técnicos que no entren en el rol de 'capacitación constante' van a sufrir los efectos de un mundo que avanza a pasos agigantados, especialmente en el campo automotriz.

La falta de aplicación con el uso del osciloscopio como herramienta fundamental de diagnóstico en talleres y concesionarios automotrices, conlleva a que se desperdicie tiempo y recursos económicos en determinar fallas específicas en motores con control electrónico, así como también molestias causadas para el propietario que pierde tiempo y dinero dejando su vehículo en un proceso de diagnóstico y reparación

\section{Materiales y Métodos}

\section{Funcionamiento del sistema de inyección CRDI}

Es esencialmente igual a la inyección multipunto de un motor de gasolina, en la cual hay un conducto común para todos los inyectores, con la diferencia de que los motores diésel trabajan a una presión más alta. Para ello se recurre a unos orificios más pequeños, dispuestos radialmente en la punta del inyector (tobera), compensando esta pequeña sección de paso con una presión mucho mayor.

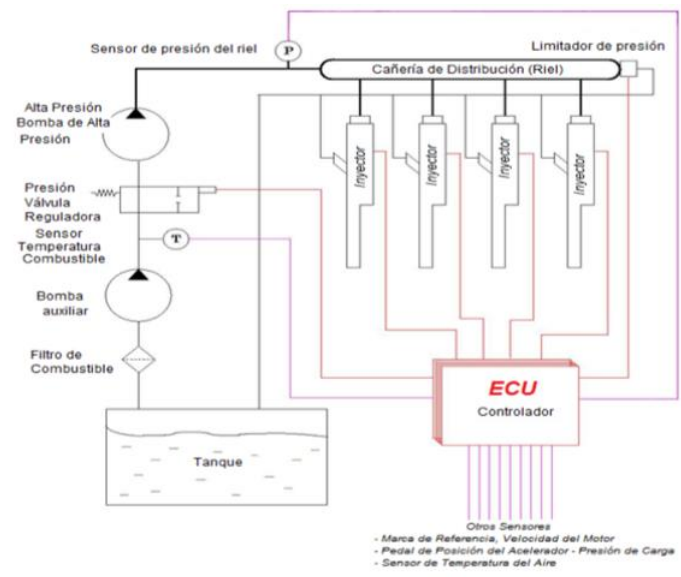

Figura 1. Esquema básico riel común 
El combustible almacenado en el tanque a baja presión, es aspirado por una bomba de transferencia que puede estar internamente en la bomba de alta presión o presurizado por una bomba eléctrica dentro del tanque de combustible, las presiones de inyección pueden variar desde unos 300 bar hasta 1600 bar, según las condiciones de funcionamiento. (Alarcón \& Jaramillo, 2007).

La bomba eleva el combustible a alta presión y transfiere a la línea de alimentación común "Riel Común”, que se distribuye a los inyectores. (Bennett, 2013).

La apertura de los inyectores es controlada por la Unidad de Control Electrónico (ECU), además mejora el funcionamiento y reduce los niveles de emisiones de gases de escape, (Riesco, Gallegos, Montefort, \& Martinez, 2005), el sistema "Riel Común” permite al motor diésel alcanzar nuevas metas en nuestro mundo siempre exigente (Figura 1).

\section{Composición del sistema de combustible}

Los componentes en el sistema de combustible (Figura 2) son diseñados para generar y distribuir alta presión, los que son controlados electrónicamente por la ECU (Unidad de Control Electrónico), en consecuencia el sistema de combustible CRDI, es completamente diferente de los sistemas de inyección diésel convencionales (Delphi, Delphi Technical training, s.f.).

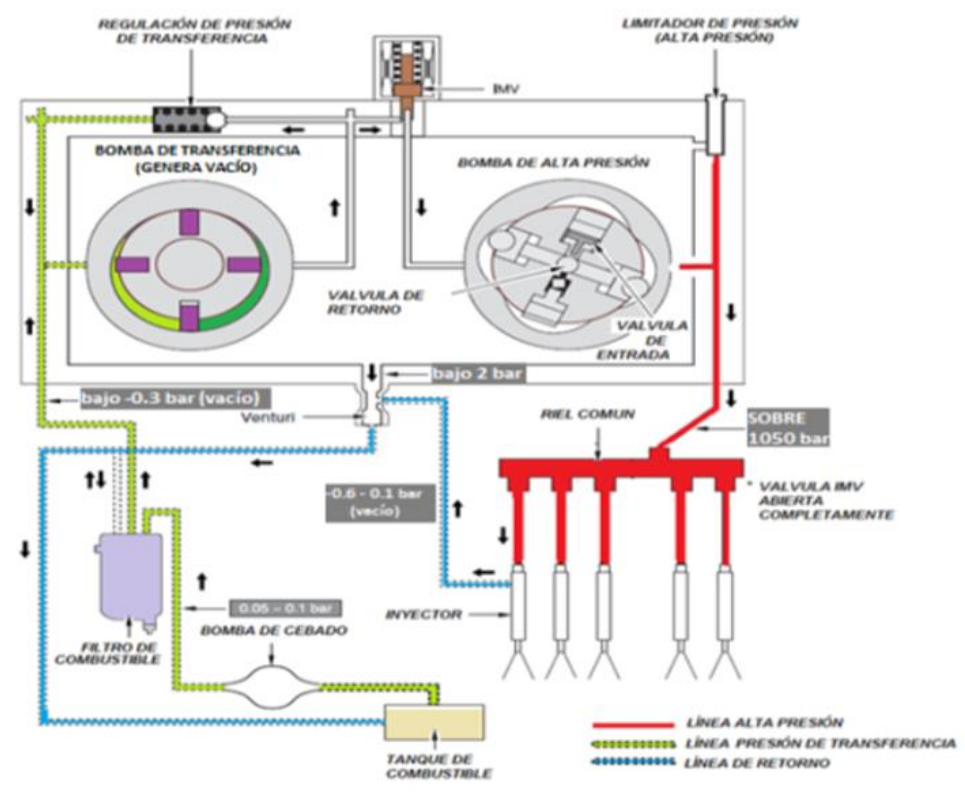

Figura 2. Circuito hidráulico de la línea de combustible (Alta presión y Transferencia)

\section{Componentes de la línea de alta presión}

En el circuito de alta presión, la bomba aumenta la presión del combustible que es enviado al riel común donde se almacena y distribuye a los inyectores. Figura 3. 


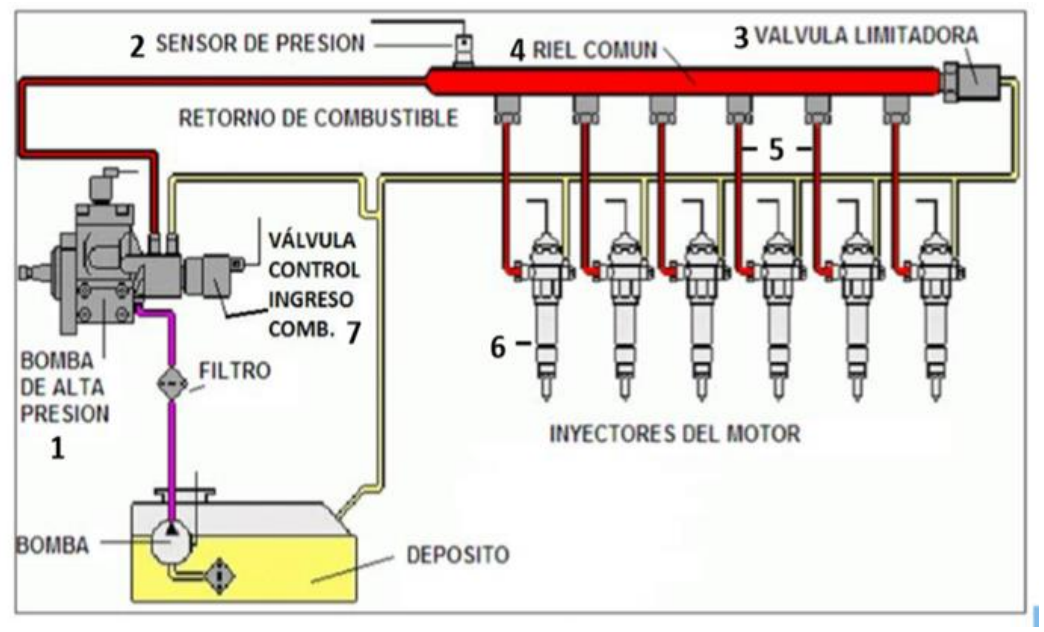

Figura 3. Circuito hidráulico de la línea de combustible de alta presión (Bomba con generador de alta presión)

Los componentes son:

1. Bomba de alta presión, las cuales pueden ser:

a) Con sistema de alta presión (Figura 2) y;

b) Con el sistema de transferencia y alta presión.

2. Sensor de presión del riel.

3. Válvula limitadora de presión.

4. Riel común.

5. Cañerías de alta presión.

6. Inyector.

7. Válvula de control de ingreso de combustible. (Inlet metering valve - IMV).

\section{Inyector}

Es un componente o elemento electrohidráulico de inyección de combustible y está compuesto por una válvula eléctrica solenoide, aguja y una tobera. La tobera del inyector se abre cuando la válvula solenoide es activada por la ECU para inyectar directamente combustible a la cámara de combustión del motor. Cuando la tobera se abre, el remanente de combustible después de la inyección retorna al tanque de combustible a través de la línea de retorno. (Delphi, Manual Common Rail Principios de Funcionamiento, 2007).

Las presiones de inyección máximas son de aproximadamente 1.600 bar. Las fuerzas que hay que superar para levantarla aguja del inyector son muy grandes. Debido a esto, es imposible controlar directamente el inyector mediante un actuador electromagnético. (Denso, 2004) El inyector está por lo tanto, indirectamente controlado por medio de una válvula que controla la presurización o la descarga de la cámara de control situado por encima de la aguja. (Figura 4). Es decir a parte del control electrónico comandado por la ECU, se requiere control hidráulico dado en diferencia de presiones. (Payri \& Desantes, Estudio de la Inyección Diesel mediante la medida del flujo de cantidad de movimiento del chorro , 2012). 


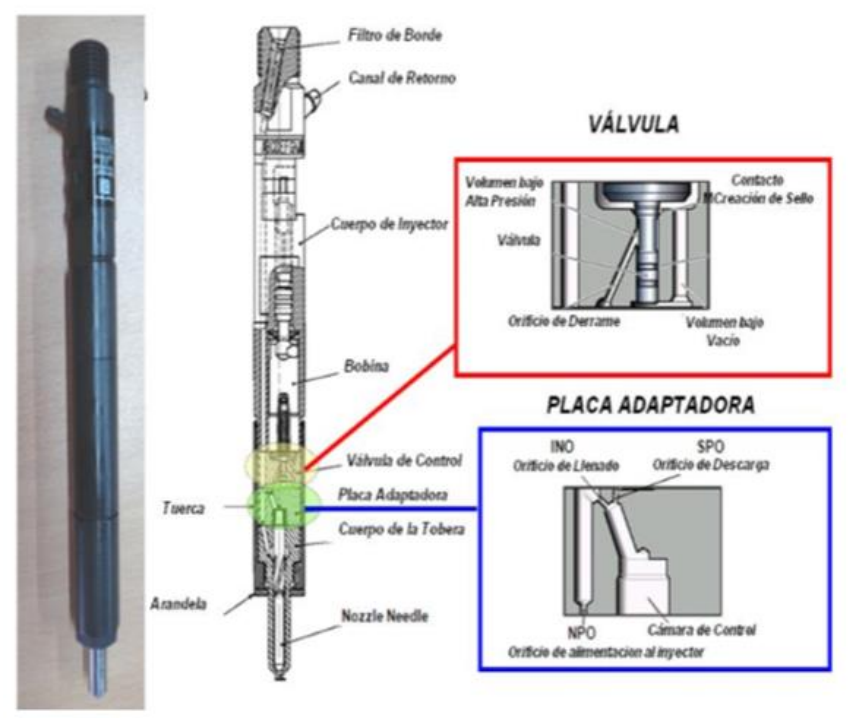

Figura 4. Componentes del inyector y conductos de combustible

\section{Control de inyección de combustible}

El control de inyección es usado para determinar las características de inyección. El control de inyección consiste en:

1. Tiempo de inyección.

2. Volumen de inyección.

3. Activación del inyector dependiendo de valores del tiempo y volumen de inyección.

a) Referencia del PMS (Punto Muerto Superior).

b) Retraso entre PMS y el comienzo del pulso Toff (Tiempo apagado).

c) Tiempo del pulso Ton (Tiempo encendido).

\section{Control del tiempo de inyección principal}

El pulso necesario para la inyección principal que está determinado en función de la velocidad del motor, carga del motor y temperatura del refrigerante.

Las correcciones están determinadas de acuerdo con la temperatura del aire, refrigerante del motor, presión atmosférica y apertura de la válvula EGR, estos parámetros ajustan el avance del tiempo de inyección disminuyendo o incrementando.

\section{Control de la inyección piloto.}

$\checkmark$ El tiempo de la inyección piloto es determinado en función de la velocidad del motor y de la cantidad de aire que ingresa al motor.

En lo que se refiere a la corrección de la inyección piloto. 
$\checkmark$ Una primera corrección se efectúa en función de la temperatura del aire que ingresa al motor y del refrigerante, esta corrección permite adaptar el caudal de la inyección piloto a la temperatura de funcionamiento del motor.

$\checkmark$ Cuando el motor está caliente el tiempo de inflamación disminuye, debido que la temperatura al final de la compresión aumenta. El caudal de la inyección piloto es reducido, debido que el ruido de la combustión es más bajo cuando el motor está caliente.

$\checkmark$ Una segunda corrección se utiliza para adaptar el caudal de la inyección piloto en función de la presión atmosférica.

\section{Control de post-inyecciones.}

Estas inyecciones situadas después de la principal tienen por objetivo calentar la línea de escape.

$\checkmark$ Se utiliza las post-inyecciones para limpiar rápidamente el catalizador.

$\checkmark$ En los vehículos equipados con un filtro de partículas, se utiliza las post- inyecciones para aumentar y estabilizar el filtro a su temperatura de regeneración. $\left(>650^{\circ} \mathrm{C}\right)$.

$\checkmark$ Las inyecciones tienen también correcciones y los factores correctivos más importantes son:

$\checkmark$ La temperatura antes del turbo.

$\checkmark$ La temperatura del catalizador.

$\checkmark \quad$ La temperatura del filtro de partículas.

\section{Pruebas preliminares}

Para el análisis de la presente investigación se toma las señales de los inyectores que varían durante el transcurso de las pruebas que se realizarán posteriormente en un vehículo determinado. Para los sensores de temperatura de refrigerante, combustible, aire y presión atmosférica se considerarán como constantes ya que su variación es despreciable porque se mantienen constante durante el tiempo de las pruebas.

Se realizan con el propósito de simular las condiciones a las cuales se someterá el vehículo y comprobar así, el correcto funcionamiento de los equipos, verificar conexiones y determinar el protocolo de pruebas que se utilizará para que sea repetible por cualquier persona.

\section{Pruebas estáticas}

Estas pruebas se las realiza con el vehículo estacionado, debe estar en posición neutral y accionado el freno de mano.Debido que las pruebas a realizar, deben cumplir con una referencia en la parte del procedimiento, antes de iniciar la prueba se crea un Protocolo de Pruebas, el mismo que sirve de patrón para que la prueba se pueda repetir las veces que sean necesarias, se ha tomado un protocolo de pruebas ya diseñado y probado, así se evita olvidos o problemas antes de empezar con las pruebas, los protocolos se realizan tanto para las pruebas estáticas como para las dinámicas, los datos obtenidos son mediante el escáner y su opción de grabado de datos (Snapshoot). (Martinez \& Robles, 2010)

A continuación, se detalla el protocolo de pruebas estáticas: 
1. Preparar todos los instrumentos de medición.

2. Confirmar la altura geográfica.

3. Confirmar la temperatura del refrigerante mayor a $80^{\circ} \mathrm{C}$.

4. Apagar el vehículo.

5. Poner la llave de encendido en posición ON.

6. Encender el vehículo y mantener en ralentí.

7. Llenar la tabla de datos para posición en ralentí.

8. Subir las revoluciones del motor a $1800 \mathrm{rpm}$ y estabilizar.

9. Llenar la tabla de datos.

10. Volver a ralentí.

11. Subir las revoluciones del motor a $2500 \mathrm{rpm}$ y estabilizar.

12. Llenar la tabla de datos.

13. Volver a ralentí.

14. Subir las revoluciones del motor a $3500 \mathrm{rpm}$ y estabilizar.

15. Llenar la tabla de datos.

16. Volver a ralentí.

17. Con el equipo osciloscopio grabar las curvas para las diferentes revoluciones del motor.

\section{Pruebas definitivas}

Se decidió que las pruebas se realizarán en dos lugares geográficos específicos: en la altura a $2300 \mathrm{msnm}$ (Figura 5) y a nivel del mar $0 \mathrm{msnm}$ (Figura 6), se realiza de esta manera para obtener un patrón de comparación y de funcionamiento de los sistemas CRDI, ya que en nuestro territorio podemos llegar a estos lugares en pocas horas, adicionalmente el parque automotor de este tipo de vehículos se encuentran distribuidos en estos lugares, como se muestra en la tabla 1.

Tabla 1. Lugares de pruebas definitivas

\begin{tabular}{|ccc|}
\hline Altura $(\mathrm{msnm})$ & Punto de Control & Lugar \\
\hline $\mathbf{2 3 0 0}$ & Llano de Jerusalén & Via San José de Minas \\
\hline $\mathbf{0}$ & Playa de Atacames & Atacames \\
\hline
\end{tabular}




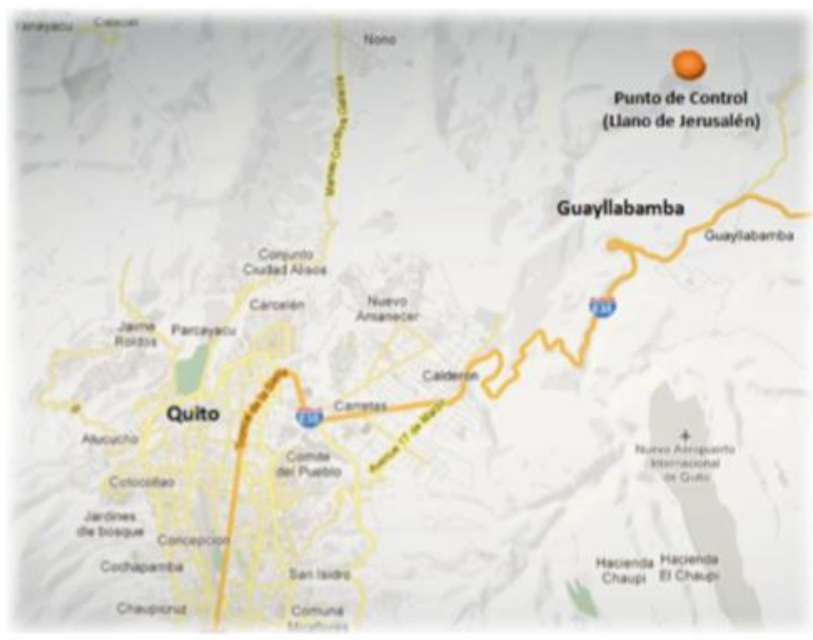

Figura 5. Punto de control 1. Llano de Jerusalén (Vía San José de Minas)

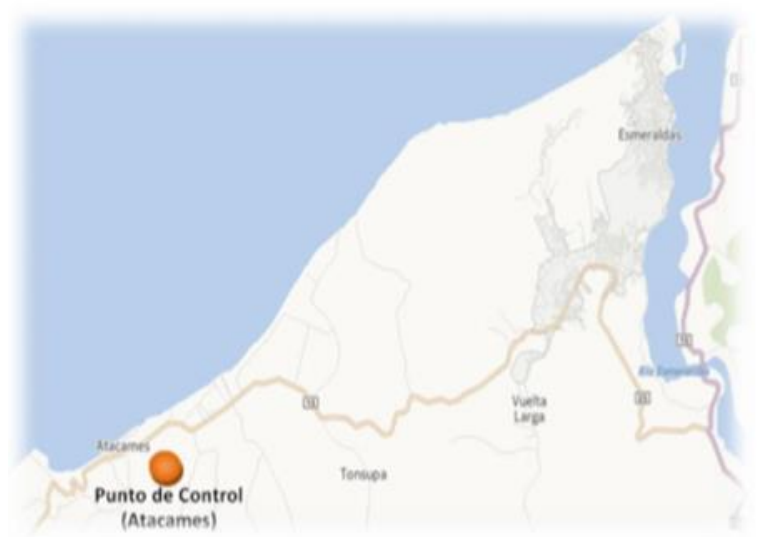

Figura 6. Punto de control 2. Playa de Atacames (Atacames)

\section{Análisis de Resultados}

\section{Planificación de pruebas}

Las carreteras en las cuales se realizarán las pruebas dinámicas al vehículo, son lugares donde la pendiente es de $6 \pm 1 \%$, este tipo de vías deben ser rectas por lo menos unos 1100 metros, para poder cumplir el ciclo de conducción propuesto.

En la Vía a San José de Minas, en el Llano de Jerusalén, se encuentra una recta aproximada de $3 \mathrm{~km}$, totalmente asfaltada, la carretera se encuentra a una altitud de $2300 \mathrm{msnm}$, se escogió horarios en los cuales la circulación de vehículos es baja, en la mañana 7 a.m. y en la tarde 2 p.m., estos fueron los horarios donde hay menor cantidad de vehículos circulando por el sector y en las cuales se puede obtener los mejores resultados en las pruebas dinámicas. 
En la carretera a Sua de la provincia de Esmeraldas, existe un camino de aproximadamente $2 \mathrm{~km}$, apropiado para realizar las pruebas, además la altitud a la que se encuentra dicha carretera es de $0 \mathrm{msnm}$, por ser una ruta de tráfico moderado las pruebas para la obtención de datos fueron a las 7 a.m., con el fin de no ocasionar molestias de las personas que transitan por el sector.

\section{Vehículo de pruebas}

El sistema de inyección Diésel Riel común significa una evolución importante a la inyección Diésel convencional, debido que trabajando a elevadas presiones y con una serie de componentes electrónicos permiten regular con gran precisión el caudal óptimo de inyección de combustible de acuerdo a la carga del motor y generando un sensible ahorro de combustible, reducción de la contaminación por combustión incompleta, mejora de rendimientos de potencia y prácticamente la eliminación del característico "ruido a Diésel".

El Ecuador está obligado a la protección del medio ambiente y las nuevas reformas gubernamentales exigen la reducción de gases contaminantes, menor consumo de combustibles, en definitiva sistemas que sean amigables con el medio ambiente, además las nuevas leyes de la Unión Europea, primero sobre la utilización de Euro 3 como límites de niveles de emisiones contaminantes y luego las Euro IV y V, significan una decisiva posición sobre nuestro país también. (Ssangyong, Manual del propietario, 2005).

Muchos de los fabricantes de vehículos livianos con tecnología CRDI han visto un buen mercado en nuestro país como marcas: asiáticas y europeas, y entre ellas están Hyundai (Terracan, Tucson), Kia (Sorento, Sportage, Carnival), Ssangyong (Rexton, Kyron (Figura 7), Korando), Mazda (BT50, TSX), Mitsubishi (L2000), Nissan (Navara), Toyota (Hilux), Volkswagen (Amarok, Touareg, Tiguan), Great Wall (H3, H5, Hover), Chevrolet (D-max), etc, es decir existen varias marcas que están presentes en nuestro país y la mayoría de ellos utilizan el sistema de inyección riel común de segunda generación y con normas Europeas Euro III, Euro IV, es decir de inyección múltiple de 1600 bar e inyectores tipo solenoide o piezo eléctricos. (Riva, 2002).

El vehículo en el cual se realizan las pruebas para la investigación es un vehículo coreano de la marca Ssangyong modelo Kyron de las siguientes características tabla 2:

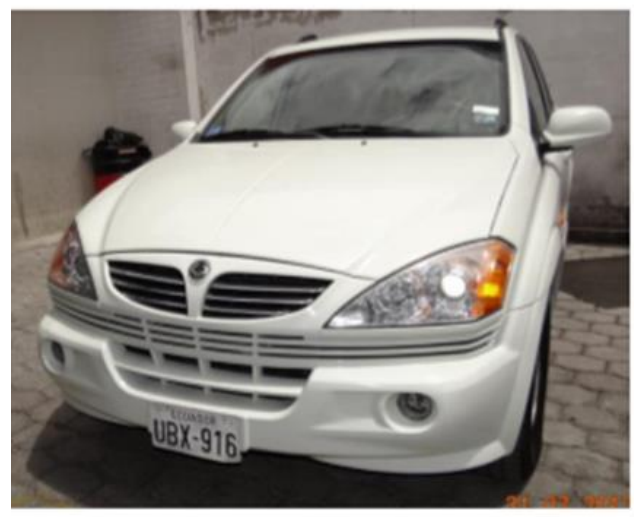


Figura 7. Ssangyong Kyron

Tabla 2. Especificaciones del vehículo de pruebas

\begin{tabular}{|l|c|}
\hline Marca & Ssangyong \\
\hline Modelo & Kyron \\
\hline Procedencia & Corea del Sur \\
\hline Año de fabricación & 2007 \\
\hline Cilindraje & $2000 \mathrm{cc}$ \\
\hline No. de cilindros & en línea \\
\hline Combustible & Diesel \\
\hline Inyección & Riel Común 2 2 $^{\text {. Generación (Delphi) }}$ \\
\hline Sobrealimentación & Turbo VGT + intercooler \\
\hline Válvulas & 16 DOHC \\
\hline Kilometraje & 95000 km \\
\hline
\end{tabular}

Se realiza ciertas modificaciones para la obtención de datos, adicionalmente el sistema de inyección es utilizado por varias marcas del mercado, los cuales permite que los parámetros que se obtienen sean aplicables y similares para dichas marcas. (Payri \& Desantes, Diagnóstico de la combustión en motores Diésel de Inyección Directa, 2012).

Para poder visualizar las ondas generadas por los diferentes sensores y actuadores del sistema CRDI, se realizó unas conexiones adicionales (figura 8) en el harness principal del motor, el cual es un grupo de cables que sale desde los sensores y actuadores del motor al ECU (Unidad de control electrónico). (Ssangyong, Manual de servicio Kyron, 2007).

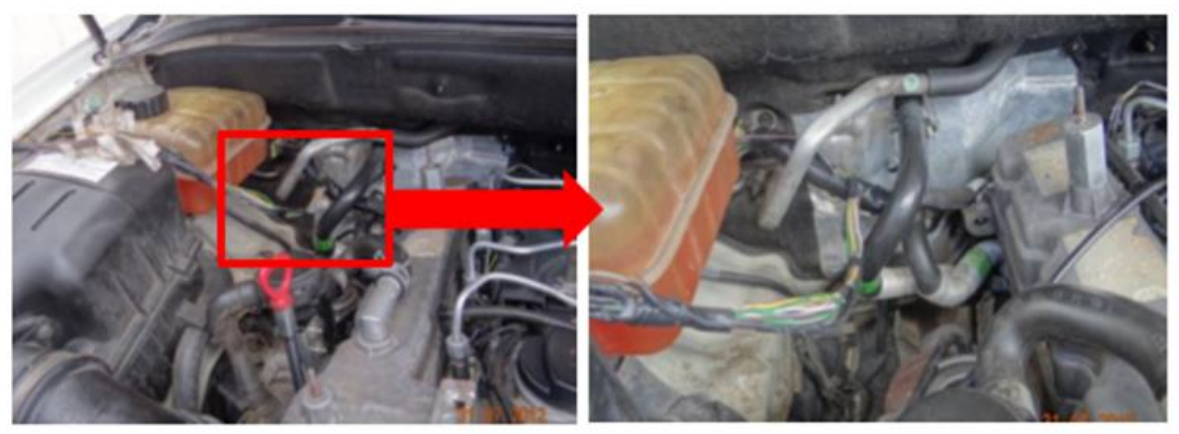

Figura 8. Conexión adicional al harness principal del motor

Adicionalmente se realiza una adaptación en la conexión de cables a una caja de control que permita de mejor manera conectar las puntas del osciloscopio y obtener los oscilogramas deseados (figura 9). (Ssangyong, Manual de servicio Kyron, 2007). 


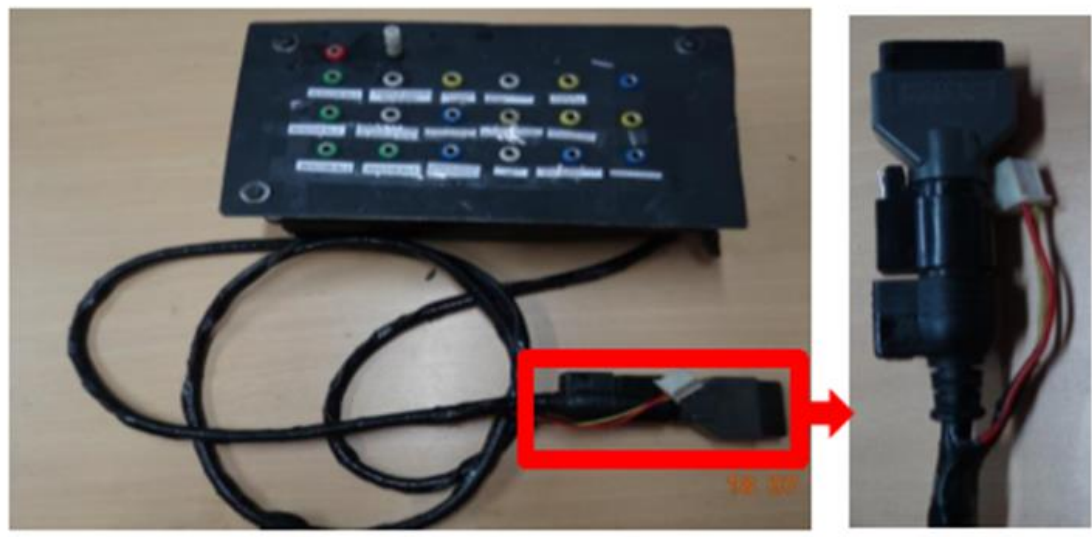

Figura 9. Caja de control para conexión del osciloscopio

\section{Altura y presión barométrica}

La medición de la altura y presión barométrica se tomará en cuenta ya que se realizarán las pruebas a nivel del mar $0 \mathrm{msnm}$ y en la altura $2300 \mathrm{msnm}$, por tanto se debe registrar la información en las tablas de datos y se requiere los cambios de los oscilogramas en dichos lugares. (Brunton, s.f.).

\section{Equipos utilizados para el desarrollo de pruebas}

Los equipos utilizados para la realización de las pruebas, tanto dinámicas como estáticas son los siguientes:

$\checkmark$ Termo higrómetro y altímetro

$\checkmark$ Celular Smartphone con GPS incorporado

$\checkmark$ Escáner de diagnóstico SCAN 100

$\checkmark$ Osciloscopio Warelead EZ-Scope M1

\section{Pruebas estáticas}

\section{Avance de la inyección principal respecto a las revoluciones.}

La (figura 10), muestra el avance de la inyección principal en grados de giro del cigüeñal dependiendo la carga del motor, en la altura muestra que los valores positivos son superiores que a nivel del mar, estos valores van en aumento con respecto a las revoluciones.

A nivel del mar algunos valores en ciertas revoluciones se muestran negativos, son grados de avance es decir antes del PMS, al contrario en la altura los valores se muestran positivos y son grados que pasaron el PMS. 


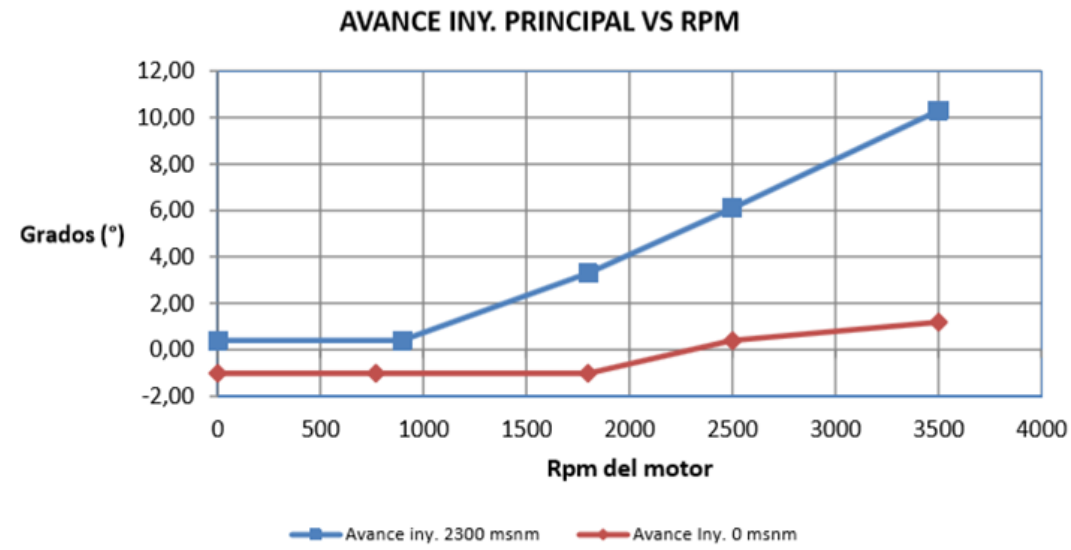

Figura 10. Avance de la inyección principal respecto a las revoluciones

\section{Avance a la inyección piloto respecto a las revoluciones.}

En la (figura 11), se muestra el avance a la inyección piloto en grados de giro del cigüeñal, en la altura y a nivel del mar son valores negativos, en este caso son grados antes del PMS y se puede notar que en la altura estos valores negativos son mayores que a nivel del mar y van en aumento con el número de revoluciones del motor. Sus valores fluctúan de $-20^{\circ}$ y $-42^{\circ}$ en la altura y a nivel del mar entre $-16^{\circ}$ y $-38^{\circ}$.

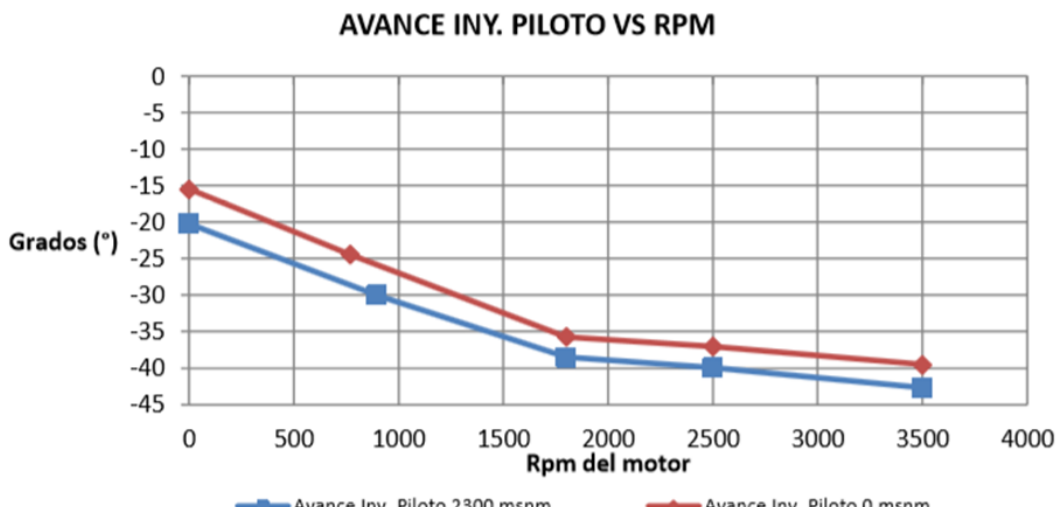

Figura 11. Avance de la inyección piloto respecto a las revoluciones

\section{Análisis de resultados}

El siguiente compilado de oscilogramas son el resumen de las pruebas estáticas realizadas a 2300 msnm y a 0 msnm Figura 12.

\section{Pruebas estáticas altura vs nivel del mar}

\section{Forma de ondas de los inyectores}




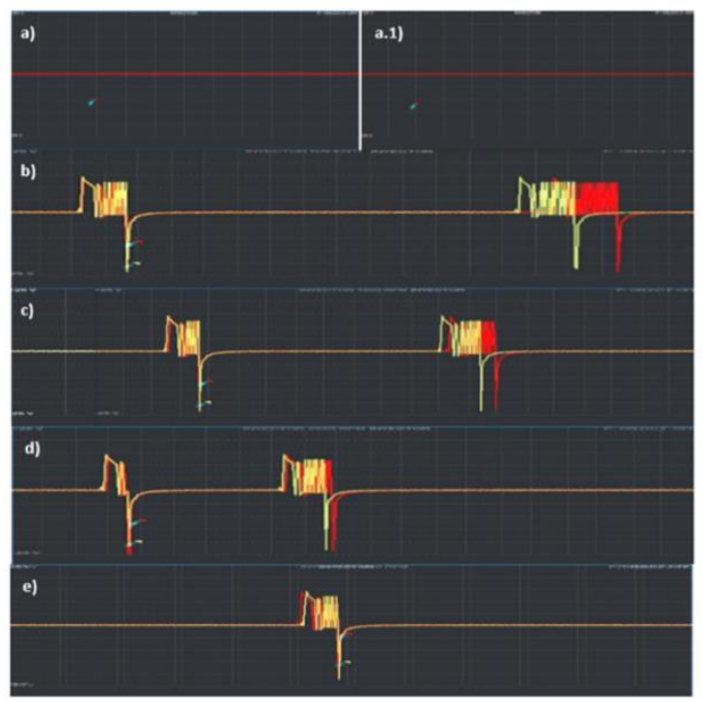

Figura 12. Forma de onda de los inyectores

a) y a.1) Formas de onda en contacto a $2300 \mathrm{msnm}$ y $0 \mathrm{msnm}$ respectivamente.

b) Ralentí. c) 1800 rpm. d) 2500 rpm. e) $3500 \mathrm{rpm}$.

Onda amarilla: $2300 \mathrm{msnm}$. Onda roja: $0 \mathrm{msnm}$

La forma de onda es obtenida del inyector No. 1. Cuando el motor del vehículo está apagado y en contacto no existe señal de voltaje y se lo muestra en la (figura 12) a) y a1), mientras tanto para las revoluciones de ralentí $(864 \mathrm{rpm}), 1800 \mathrm{rpm}, 2500 \mathrm{rpm}, 3500 \mathrm{rpm}$ el inyector genera un voltaje pico máximo promedio de $14 \mathrm{~V}$ positivos y un pico máximo negativo de $24 \mathrm{~V}$ en las dos condiciones de medición en la altura $(2300 \mathrm{msnm})$ y a nivel del mar (0 msnm). (Tabla 3).

Tabla 3. Pulso de inyección y voltaje de inyectores.

\begin{tabular}{|c|c|c|c|c|c|c|c|c|}
\hline \multicolumn{9}{|c|}{ MEDICIÓN DEL PULSO DE LA PRE-INYECCIÓN Y PRINCIPAL } \\
\hline \multirow{2}{*}{ DESCRIPCIÓN } & \multicolumn{2}{|c|}{ RALENTi } & \multicolumn{2}{|c|}{1800} & \multicolumn{2}{|c|}{2500} & \multicolumn{2}{|c|}{3500} \\
\hline & $\begin{array}{c}0 \\
\mathrm{msnm}\end{array}$ & $\begin{array}{c}2300 \\
\mathrm{msnm}\end{array}$ & $0 \mathrm{~m}$ & $\begin{array}{c}2300 \\
\mathrm{msnm}\end{array}$ & $0 \mathrm{n}$ & $\begin{array}{r}2300 \\
\mathrm{msnm}\end{array}$ & $\mathrm{nm}$ & $2300 \mathrm{msnm}$ \\
\hline PRE-INYECCIÓN (ms) & 0,52 & 0,54 & 0,43 & 0,45 & 0,34 & 0,35 & N/A & N/A \\
\hline $\begin{array}{l}\text { INYECCIÓN PRINCIPAL } \\
\text { (ms) }\end{array}$ & 0,73 & 0,86 & 0,56 & 0,55 & 0,49 & 0,5 & 0,53 & 0,51 \\
\hline
\end{tabular}

\begin{tabular}{|c|c|c|c|c|c|c|c|c|}
\hline \multicolumn{9}{|c|}{ VALORES DE VOLTAJE } \\
\hline $\operatorname{ESTADO}(\mathrm{rpm})$ & \multicolumn{2}{|c|}{ RALENTI } & \multicolumn{2}{|c|}{1800} & \multicolumn{2}{|c|}{2500} & \multicolumn{2}{|c|}{3500} \\
\hline DESCRIPCIÓN & $0 \mathrm{msnm}$ & $\begin{array}{r}2300 \\
\mathrm{msnm}\end{array}$ & $0 \mathrm{msnm}$ & $\begin{array}{r}2300 \\
\mathrm{msnm}\end{array}$ & $0 \mathrm{msnm}$ & $\begin{array}{r}2300 \\
\mathrm{msnm}\end{array}$ & $\begin{array}{c}0 \\
\text { msnm }\end{array}$ & $\begin{array}{r}2300 \\
\mathrm{msnm}\end{array}$ \\
\hline VOLTAJE MÁXIMO (V) & 14,4 & 14,4 & 14 & 14,4 & 14,05 & 14,5 & 14,05 & 14,4 \\
\hline VOLTAJE MINIMO (V) & $-24,35$ & $-21,25$ & $-23,15$ & -24 & $-25,6$ & $-22,5$ & $-23,4$ & $-22,5$ \\
\hline
\end{tabular}




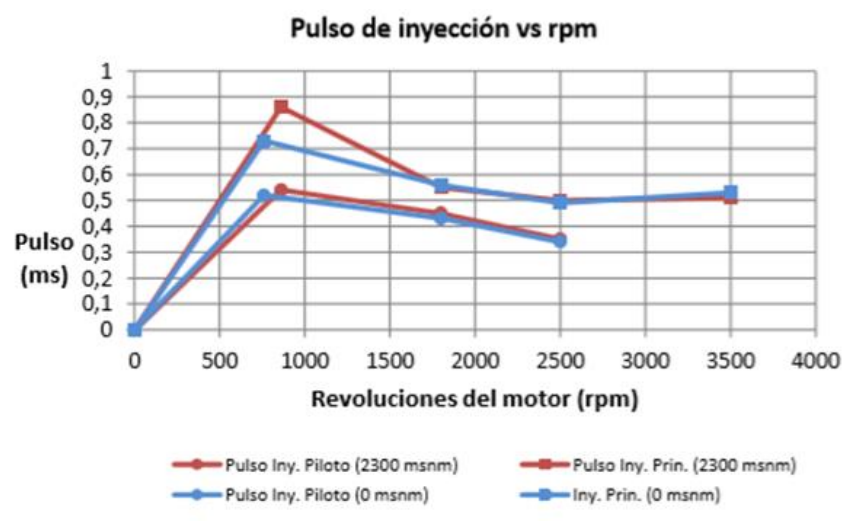

Figura 13. Pulso de inyección respecto a las rpm

Con respecto al pulso de inyección, es el tiempo que el inyector se abre para dejar pasar combustible en la inyección piloto y la principal, los pulsos son mayores cuando están en ralentí, pero van disminuyendo al aumentar las revoluciones, este efecto se presenta tanto en la altura como a nivel del mar, cabe mencionar que los pulsos son los mismos, donde existe una variación es en el adelanto y retraso del tiempo de inyección por efecto de la variación de altura como se muestran en las figuras subsecuentes b, c, d y e. Una característica adicional, donde se puede notar una pequeña variación es en el pulso de las revoluciones de ralentí (figura 13).

\section{Conclusiones}

Los oscilogramas de los sensores y actuadores obtenidos del motor CRDI son de gran importancia para realizar los diagnósticos en este tipo de motores ya que inciden en el rendimiento del motor.

La variación de altitud incide en los sensores y actuadores del motor CRDI, sin embargo toda la gestión del control electrónico que actúa en el motor tiene un efecto en el cual el conductor no siente una pérdida del rendimiento en el vehículo cuando éste se encuentra en la altura.

El análisis de cada uno de los actuadores del CRDI muestra claramente su funcionamiento y comportamiento frente al número de revoluciones y altura determinada.

\section{Bibliografía}

Alarcón, C., \& Jaramillo, J. (2007). Guía para el diagnóstico del sistema de inyección CRDI Bosch. Cuenca: Universidad Salesiana.

Bennett, S. (2013). Medium/Heavy Duty Truck Engines, Fuel \& Computerized Management Systems. EEUU.

Brunton. (s.f.). Manual del propietario Termo Hidrógeno y altímetro. Obtenido de https://www.brunton.com/products/adc-pro 
Delphi. (2007). Manual Common Rail Principios de Funcionamiento. Francia: Delphi France SAS.

Delphi. (s.f.). Delphi Technical training . Obtenido de https://www.delphiautoparts.com/sites/default/files/2018_training_prospectus_external_p rint.pdf

Denso. (2004). Service Manual. Japon: Senso Corporation.

Martinez, J., \& Robles, F. (2010). Estudio del comportamiento de las variables de un motor de inyección electrónica respecto a la altura sobre el nivel del mar. Quito: Tesis EPN.

Payri, F., \& Desantes, F. (2012). Diagnóstico de la combustión en motores Diesel de Inyección Directa. Barcelona: Reverté.

Payri, F., \& Desantes, J. (2012). Estudio de la Inyección Diesel mediante la medida del flujo de cantidad de movimiento del chorro. Barcelona: Reverté.

Riesco, J., Gallegos, A., Montefort, J., \& Martinez, S. (2005). Procesos Alternativo de Combustión en Motores de Combustión Interna. Guanajuato: Universidad de Guanajuato.

Riva, C. (2002). Diseño concurrente. Cataluña: Edicions UPC.

Ssangyong. (2005). Manual del propietario. Corea: Ssangyong Motor Company.

Ssangyong. (2007). Manual de servicio Kyron. Corea: Ssangyong Motor Company.

Ssangyong motor co., 1. (2008). Manual de Taller Rexton. Corea: Ssangyong Motor company.

Van, D., \& Meyer, W. (1981). Manual de Técnica de la investigación Educacional. Barcelona: Paidos Ibérica. 